\title{
Ocorrência de flebotomíneos (Diptera: Psychodidae: Phlebotominae) no ambiente peridomiciliar em área de foco de transmissão de leishmaniose tegumentar no município de Manaus, Amazonas
}

Sônia Rolim REIS ${ }^{1}$, Luis Henrique Monteiro GOMES², Natália Mota FERREIRA³ ${ }^{3}$ Liliane da Rocha NERY , Francimeire Gomes PINHEIRO ${ }^{5}$, Luanda de Paula FIGUEIRA ${ }^{6}$, Fabiane Veloso SOARES ${ }^{7}$, Antonia Maria Ramos FRANCO ${ }^{8}$

\section{RESUMO}

A leishmaniose é uma antropozoonose com focos naturais, nos quais a infecção é mantida em ecótopos silvestres entre a população de vetores e seus hospedeiros vertebrados. Neste estudo foram identificadas espécies de flebotomíneos envolvidas na transmissão de leishmaniose tegumentar no Bairro Cidade de Deus, onde há focos da doença humana e animal. O bairro está situado à margem da reserva florestal Adolpho Ducke. Foram capturados 122 espécimens em épocas variadas, utilizando-se armadilhas luminosas do tipo CDC. As espécies de flebotomíneos mais encontradas foram Lutzomyia umbratilis, L. rorotaensis e L. olmeca nociva. Nas proximidades de uma das residências onde foram realizadas as coletas de flebotomíneos, verificouse descarte de resíduos orgânicos (lixo), o que possibilita a atração de reservatórios silvestres presentes na reserva florestal favorecendo a ocorrência do ciclo de transmissáo da doença no peridomicílio.

PALAVRAS-CHAVE: Manaus, Leishmania, vetores.

\section{Occurrence of sandflies (Diptera: Psychodidae: Phlebotominae) in the peridomestic environment in an area of transmission focus for cutaneous leishmaniasis in Manaus, Amazon}

\begin{abstract}
Leishmaniasis is an antropozoonosis with natural foci, in which infection is maintained in wild ecotopes among the population of vectors and their vertebrate hosts. This study identified sand fly species involved in transmission of leishmaniasis in the east area of Manaus, in households in the neighborhood of Cidade de Deus, where there are outbreaks of human and animal disease. The vicinity is near to Adolpho Ducke forest reserve. In the study, 122 specimens were captured at different times, using the $C D C$ light traps. The most commonly encountered species of phlebotomine sandflies were Lutzomyia umbratilis, L. rorotaensis and L. olmeca nociva. Near one of the residences where the collections were made, there was disposal of organic waste, a fact that favors the attraction of animal reservoirs in the forest reserve, thus enabling the occurrence of the disease transmission cycle in the peridomicile.
\end{abstract}

KEYWORDS: Manaus, Leishmania, vectors

\footnotetext{
${ }^{1}$ Coordenação de Pesquisas em Sociedade, Ambiente e Saúde, Instituto Nacional de Pesquisas da Amazônia - INPA. Av. André Araújo 2936, Aleixo, Manaus, AM. Telefone: (92) 3643 3065, FAX (92) 3643 3068. E-mail: reis@inpa.gov.br.

2 Instituto Nacional de Pesquisas da Amazônia. Email: luis@inpa.gov.br

3 Email: natalia.mf22@hotmail.com

4 Instituto Nacional de Pesquisas da Amazônia. Email: lilianecr76@gmail.com

${ }^{5}$ Universidade Federal do Amazonas. Email: meireg@inpa.gov.br

${ }^{6}$ Universidade Federal do Amazonas. Email: luandafigueira@yahoo.com.br

7 Universidade Federal do Amazonas. Email: fabianepinks@hotmail.com

8 Instituto Nacional de Pesquisas da Amazônia. Email: afranco@inpa.gov.br
} 
A leishmaniose é uma antropozoonose com focos naturais, nos quais o parasito é mantido em ecótopos silvestres entre a população de vetores e seus hospedeiros vertebrados. Nas Américas, são atualmente reconhecidas 11 espécies dermotrópicas de Leishmania (Kinetoplastida: Trypanosomatidae) causadoras de doença humana e oito espécies descritas somente em animais. O gênero Lutzomyia (Diptera: Psychodidae: Phlebotominae) é considerado o de maior importância por conter espécies transmissoras de patógenos aos humanos, com ampla distribuiçáo no Brasil desde regióes do sul até o norte do país. As principais espécies envolvidas na transmissão da leishmaniose Tegumentar Americana (LTA) são: Lutzomyia flaviscutellata, L. whitmani, L. umbratilis, L. intermedia, L. wellcomei e L. migonei (Brasil 2007; Freitas et al. 2002; Silveira et al. 2002).

Estas espécies de flebotomíneos foram incriminadas como vetoras por atenderem aos critérios que atribuem a uma espécie a competência vetorial.

Os critérios sugeridos para incriminar efetivamente uma determinada espécie de flebotomíneo como vetora de leishmaniose são: antropofilia; distribuição espacial em concordância com a ocorrência dos casos de infecção humana; infecção natural por parasitos identificados como pertencentes à mesma espécie de Leishmania que infecta o homem; atração por mamíferos reservatórios de Leishmania e a prova conclusiva de incriminação vetorial seria a capacidade desses flebotomíneos de se infectarem e transmitirem experimentalmente o parasito, através da picada, de hamster para hamster (Mesocricetus auratus) em laboratório. A relação entre o vetor e o parasito é espécie - específica, verificando-se uma íntima relação entre estes elos epidemiológicos (KillickKendrick 1990).

A epidemiologia da leishmaniose é extremamente complexa e pode ser alterada pela ocorrência de mudanças em qualquer posição no ciclo: vetor-reservatório-homem. $\mathrm{O}$ desmatamento e a urbanização são exemplos de alteraçóes que são seguidas por mudanças principalmente, em reservatórios e na população de insetos vetores (Pinheiro et al. 2010; Silva et al. 2010). No Brasil, já foram identificadas seis espécies do subgênero Viannia e uma do subgênero Leishmania. As principais são: $L$. (V.) braziliensis, $L$. (V.) guyanensis e $L$. (L.) amazonensis e, mais recentemente, $L$. (V.) lainsoni, $L$. (V.) naiff, $L$. (V.) lindenbergi e $L$. (V.) shawi encontradas em estados das regiôes Norte e Nordeste (Silveira et al. 2002; Brasil 2007). Até o momento, no Estado do Amazonas, a infecção ocorre no homem pelas espécies Leishmania (L.) amazonensis, $L$. (V.) braziliensis, L. (V.) guyanensis e $L$. (V.) naiffi (Reis e Franco 2010).

As modificaçóes ambientais que vem ocorrendo no município de Manaus podem acarretar diferentes adaptaçóes e mudanças quanto ao padrão epidemiológico da LTA. Estas prováveis mudanças poderiam eventualmente possibilitar a ocorrência de outros ciclos de transmissão, nos quais os homens e os animais domésticos estariam envolvidos (Brasil 2007; Reis 2008). Neste contexto, identificar a circulação de espécies de flebotomíneos em áreas de foco de transmissáo de LTA humana e animal é de extrema importância, principalmente em ambientes peridomiciliares onde o contato do vetor com o hospedeiro vertebrado é mais intenso e não ocasional.

Neste estudo, foram realizadas capturas de flebotomíneos em residências na cidade de Manaus próximo à reserva florestal Adolpho Ducke (RFAD), área considerada de foco de LTA. Foram selecionados domicílios onde havia casos de LT humana e animal, confirmados por estudos anteriores no bairro Cidade de Deus (Reis, 2008). As residências estão localizadas à margem da RFAD, área de mata primária. Na captura dos insetos, utilizou-se armadilha luminosa do tipo "CDC modificada" (Modified CDC miniature - HausherrMachine Works, New Jersey, EUA). As armadilhas foram penduradas com auxílio de barbante nos galhos das árvores a aproximadamente um metro de altura do solo durante o período crepuscular e noturno das 17 às 8 horas do dia seguinte (Pinheiro et al. 2010). Foram realizadas nove coletas utilizando seis armadilhas em cada uma delas no peridomicílio de duas residências nos períodos de Agosto e Setembro/2009; Janeiro, Março e Maio/2010. Os insetos foram triados e as fêmeas dissecadas no laboratório de leishmaniose e Doença de Chagas do Instituto Nacional de Pesquisas da Amazônia (INPA).

Um total de 122 flebotomíneos, sendo 38,5\% (47) machos e $61,5 \%$ (75) fêmeas, foram capturados. Destes, considerando apenas as fêmeas, dez diferentes espécies (Tabela 01) foram identificadas sendo as espécies mais abundantes: Lutzomyia umbratilis - 30,6\% (23), Lu. rorotaensis - 25,3\% (19) e

Tabela 1 - Número de fêmeas capturadas próximo as duas residências no período de outubro/2009 a maio/2010, no bairro Cidade de Deus, Manaus, AM, Brasil.

\begin{tabular}{lcc}
\hline \multicolumn{1}{c}{ Espécie } & $\begin{array}{c}\text { Fêmeas } \\
\text { Dissecadas }\end{array}$ & $\begin{array}{c}\text { Infecção } \\
(+/-)\end{array}$ \\
\hline Lutzomyia umbratilis & 23 & - \\
L. rorotaensis & 19 & - \\
L. olmeca nociva & 18 & - \\
L. dendrophyla & 4 & - \\
L. flaviscutellata & 2 & - \\
L. davisi & 2 & - \\
L. georgii & 4 & - \\
L. derelicta & 1 & - \\
L. anduzei & 1 & - \\
L. infraespinosa & 1 & - \\
Total & 75 & - \\
\hline
\end{tabular}


Lu. olmeca nociva - 24\% (18), vetores incriminados na transmissão de L. (V.) guyanensis, L. (V.) naiff e L. (L.) amazonensis, respectivamente (Lainson et al.1990; Freitas et al. 2002) .

Dentre as espécies de flebotomíneos coletadas e identificadas próximo às residências selecionadas, L. umbratilis, L. olmeca nociva e L. flaviscutellata são importantes transmissoras de LTA (Freitas et al. 2002; Silva et al. 2007).

Como a taxa de infecção natural por tripanossomatídeos em flebotomíneos é em torno de $1 \%$, e neste estudo foram realizadas apenas nove coletas perfazendo um total de 75 fêmeas capturadas e negativas para a presença de infecção, mais estudos direcionados à entomofauna local em ambientes antropizados se fazem necessários a fim de se identificar a presença de espécimens vetores infectados e a circulação do parasito nestas áreas.

$\mathrm{Na}$ região Amazônica, o gênero Lutzomyia apresenta variável importância médica; dentre suas espécies podemos citar: L. ayrozai e L. rorotaensis infectadas com L. (Viannia) naiff (Lainson et al. 1990; Freitas et al. 2002; Gil et al. 2003; Silva et al. 2007). No presente estudo, L. rorotaensis foi à segunda espécie mais coletada próximo às residências.

A probabilidade de transmissão da doença depende das condições de exposição de humanos e de animais domésticos aos flebotomíneos (Guerra et al. 2006). Na proximidade de uma das duas residências onde foram realizadas as coletas, verificou-se descarte de resíduo orgânico, fato que atrai reservatórios silvestres presentes na reserva, favorecendo o ciclo de transmissão da LTA no peridomicílio. Desta forma, constitui-se motivo de alerta a predominância destas espécies nestas áreas, o que implica na possibilidade de surtos de LTA.

\section{BIBLIOGRAFIA CITADA}

Brasil 2007. Ministério da Saúde. Fundação Nacional de Saúde. Manual de controle da leishmaniose tegumentar americana. $2^{\circ}$ Ed.Brasilia. 182 pp.

Freitas, R.A.; Naiff, R.D. Barret,T.V. 2002. Species diversity and flagellate infections in the Sandy fly near Porto Grande, State of Amapá, Brazil (Diptera: Psychodidae Kinetoplastida: Trypanosomatidae). Memórias do Instituto Oswaldo Cruz, 97 (1):53-59.
Gil, L.H.S; Basano S.A.; Souza, A.A.; Silva, M.G.S.; Barata I.;Ishikawa E.A.; Camargo L.M.A.; Shaw J.J. 2003. Recent Observations on the Sand Fly (Diptera: Psychodidae) Fauna of the State of Rondônia, Western Amazônia, Brazil: the Importance of Psychdopygus davisi as a Vector of Zoonotic Cutaneous Leishmaniasis. Memórias do Instituto Oswaldo Cruz, 98(6): 751-755.

Guerra, J. A.O.; Ribeiro, J.A.S.; Coelho, L.I.A.R. C.; Barbosa, M.G.V.; Paes, G.P. 2006. Epidemiologia da Leishmaniose Tegumentar na comunidade São João, Manaus, Amazonas, Brasil. Caderno de Saúde Pública, 22: 2319-27.

Killick-Kendrick, R. 1990. Phlebotomine vectors of leishmaniasis: a review. Medical and Veterinary Entomology, 4: 1-24.

Lainson,R.; Shaw,J.J.; Silveira, F.T.; Ishikawa,E.A.Y. 1990. Cutaneous leishmaniasis of man due to Leishmania (Viannia) naïff Lainson and Shaw, 1989. Annales de Parasitologie Humaine et Comparee, 65: 282-284.

Pinheiro, F.G.; Freitas, R.A.; Rocha, L.C.; Franco, A.M.R. 2010. Primeiro registro de Lutzomya (Trichopygomya) convitii Ramirez Perez, Martins \& Ramirez (Diptera: Psychodidae) no Brasil. Neotropical Entomology, 39: 676 - 677.

Reis, S.R. 2008. Diagnóstico biológico e molecular da Leishmaniose Tegumentar Americana em cães domésticos (Canis familiaris) no municipio de Manaus, AM, BR. Tese de Doutorado em Biotecnologia. Universidade Federal do Amazonas, Manaus, Amazonas. 96 pp (in portuguese)

Reis, S.R.; Franco A.M.R. 2010. A Leishmaniose Tegumentar Americana no Estado do Amazonas, Brasil: Aspectos epidemiológicos da Leishmaniose canina. Revista Conselho Federal de Medicina Veterinária, 50: 35-40.

Silva, D. F.; Freitas, R. A.; Franco, A. M. R. 2007. Diversidade e Abundância de Flebotomíneos do Gênero Lutzomyia (Diptera: Psychodidae) em Áreas de Mata do Nordeste de Manacapuru, AM. Neotropical Entomology, 36: 138-144.

Silveira, F.T.; Ishikawa, E.A.Y.; De Souza, A.A.A.; Lainson, R. 2002. An outbreak of cutaneous leishmaniasis among soldiers in Belém, Pará State, Brazil caused by Leishmania (Viannia) lindenbergin. sp., a new leishmanial parasite of man in the Amazon region. Parasitology, 85: 43-50.

Recebido em: 07-10-2011

Aceito em: 05-04-2012 
